Systematic Review

\title{
High Prevalence of Neuropathic Pain Component in Patients with Low Back Pain: Evidence from Meta-Analysis
}

Kapil Gudala, PhD Scholar ${ }^{1}$, Dipika Bansal, MD, DM ${ }^{1}$, Rambabu Vatte, MPharm¹, Babita Ghai, MD², Fabrizio Schifano, FRCPsych², and Chandrasehkar Boya, PhD Scholar ${ }^{1}$

From: ${ }^{\text {NNational Institute of }}$

Pharmaceutical Education and Research, Mohali, Punjab, India; ${ }^{2}$ Postgraduate Institute of Medical Education \& Research, Chandigarh, India

Address Correspondence: Dipika Bansal, MD, DM National Institute of Pharmaceutical Education and Research, Mohali 160062, Punjab, India Email:

dipikabansalo79@gmail.com

Disclaimer: There was no external funding in the preparation of this manuscript. Conflict of interest: Each author certifies that he or she, or a member of his or her immediate

family, has no commercial association (i.e., consultancies, stock ownership, equity interest, patent/licensing arrangements, etc.) that might pose a conflict of interest in connection with the submitted manuscript.

Manuscript received: 06-12-2016 Revised manuscript received: 12-05-2016

Accepted for publication: 12-14-2016

Free full manuscript: www.painphysicianjournal.com
Background: Low back pain (LBP) is a complex syndrome which includes a nociceptive (NcP) component, a neuropathic (NeP) component, or a mixture of components (mixed pain). The NeP component $(\mathrm{NePC})$ in LBP is defined as the presence of NeP with or without an NcP.

Objective: This meta-analysis aimed at assessing the pooled prevalence of NePC in patients with LBP and at identifying the factors causing significant heterogeneity in reported prevalence.

\section{Study Design: Meta-analysis}

Methods: A systematic literature search was carried out, with inclusion of all epidemiological studies describing the NeP prevalence levels in LBP patients while using standard diagnostic methods. The "pooled prevalence rate (PPR)" of NePC, either on its own or in combination with NcP, was calculated. A pre-specified subgroup analysis was carried out, considering LBP duration, presence of leg pain, diagnostic method(s), and questionnaire(s) used.

Results: The meta-analysis included 20 studies relating to a total of 14,269 LBP patients, of whom 7,969 patients (55.8\%) were identified as presenting with NePC. The pooled PR (95\% $\mathrm{Cl})$ of NePC in patients with LBP was $0.47(0.40-0.54)$, while the pooled PR of NcP was 0.56 $(0.48-0.63)$. Higher NePC pooled PR values were identified in LBP with leg pain as compared to uncomplicated LBP (respectively: $0.60 ; 0.47-0.73$ vs $0.27 ; 0.23-0.31 ; P_{\text {interaction }}<0.01$ ).

Limitations: The quality of the included studies was assessed using ad-hoc criteria. Due to the limited number of available studies, one may need to be cautious in reaching conclusions about the impact of disease duration on NePC prevalence values. We pooled studies which used a range of different diagnostic methods, with putatively different sensitivity/specificity diagnosing levels.

Conclusion: Overall, high NePC prevalence levels were here identified in LBP patients. As the pain is a subjective phenomenon and there is no gold standard for the diagnosis of NePC, there is the possibility that the pooled effect estimate may alter depending upon the diagnostic method used.

Key words: Neuropathic pain, nociceptive pain, low back pain, symptom-based questionnaire, chronicity

Pain Physician 2017; 20:343-352 ow back pain (LBP) is the most frequently observed chronic pain condition (1). It may be associated with leg pain and/or motor/ sensory/reflex deficits in the nerve root distribution areas. Thus, LBP can present with nociceptive (NcP), neuropathic (NeP), or mixed pain components (mixed pain) (1). The neuropathic pain component (NePC) in LBP is defined as the presence of NeP either on its own or associated with NcP. Recently, the development of multiple measurement scales, such as the 
painDETECT questionnaire (PDQ) (2), the neuropathic pain questionnaire (NPQ) (3), the Leeds Assessment of Neuropathic Symptoms and Signs (LANSS) (4), the ID-pain (5), the Douleur neuropathique 4 (DN4) (6), and the standardized evaluation of pain (StEP) (7) have facilitated a better identification of NePC in LBP conditions. The extent of involvement of NePC in LBP patients is extensively debated, with reported prevalence rates being in the range of $28.1 \%$ to $71.2 \%$ $(8,9)$. Recognition of NePC is important, since it may significantly affect the patient's psychological and social functioning, while placing considerable financial demands on society (10).

Categorizing LBP as either NcP or NeP is not always straightforward (11). A range of different approaches have been proposed to facilitate this distinction, including physician's judgement, International Association for the Study of Pain (IASP) Treed's criteria (12), different questionnaires (2-7), and finally quantitative sensory (QST) and pharmacological testing methods (13). Identifying the presence of NePC in LBP is important, since this may present as a challenging treatment/management issue (14), typically associated with low rates of treatment success (15). Patients with acute LBP may show NeP symptoms similar to those of chronic LBP (CLBP), with lack of recognition possibly leading to suboptimal drug dosages being administered, with the risk that the acute pain condition may persist and become subacute/ chronic $(16,17)$.

The current meta-analysis is aimed at assessing the pooled prevalence of NePC in patients with acute, subacute, and chronic LBP and identifying the factors causing significant heterogeneity in reported prevalence.

\section{Methods}

\section{Search Strategy}

Two electronic databases (PubMed and EMBASE, from their inception until August 2015) were searched independently by 2 authors without any language restriction. Both full texts and $\mathrm{MeSH}$ terms were considered to identify those epidemiological (e.g., case control and cohort) studies focusing on assessing NeP prevalence levels in LBP patients. Searches were conducted using synonyms and combinations of the following search terms: "neuropathic pain," "prevalence," "low back pain," and "leg pain." In addition, related systematic reviews and selected studies' reference lists were screened for any other relevant study to be included in the present analysis.

\section{Study Selection Criteria}

Two authors independently reviewed the titles and abstracts of all identified citations as per inclusion and exclusion criteria. An abstract was considered to be relevant if it reported original data, originated from epidemiological studies, and reported the prevalence of NePC in patients with LBP. For the present analysis, patients were classified in 3 groups: a) patients with exclusive NcP, b) patients with exclusive $\mathrm{NeP}$, and c) patients with mixed pain (e.g., suffering from both NeP and NcP). NePC was here defined as presence of NeP symptoms exclusively and/or associated with NcP symptoms.

Studies were excluded if data regarding prevalence of $\mathrm{NeP}$ in LBP patients were missing/not clearly reported, and when diagnostic methods were either not standardized or not reported. Single case reports, comments, letters, editorials, protocols, guidelines, and review papers were excluded. When there were multiple publications for the same population, only the most detailed report was included. Any discrepancies were resolved by a consensus conference.

\section{Data Extraction and Synthesis}

Two authors independently extracted a range of relevant information, including first author name, year of publication, country of patients' recruitment, study setting, study design, sample size, diagnostic methods used to assess NePC, NeP/NePC reported prevalence rates (PR) and $95 \%$ confidence interval $(\mathrm{Cl})$, duration of disease, and definitions of NeP, NcP, and NePC. If unavailable, both $\mathrm{PR}$ and $95 \% \mathrm{Cl}$ of $\mathrm{NeP} / \mathrm{NePC}$ were calculated from the raw data available. If a study reported PRs of NeP and mixed pain separately, they were pooled together to get an estimate of NePC.

\section{Quality Assessment}

Consistent with the approach of both Hoogendoorn et al (18) and De Vet et al (19), all included studies were independently assessed for their methodological quality by 2 authors. These researchers had developed and tested a list of 23 criteria to be used to assess the methodological quality of prospective, cohort, and case control studies. Out of this list of criteria, Fishbain et al (20) selected 10 criteria that were deemed appropriate for the assessment of NeP prevalence in LBP. To this list, Fishbain et al (20) have added one extra criterion. We have excluded 5 questions and added one (relating to the characteristics of recruitment, e.g., consecutive or not) question for this study, resulting in a total of 7 criteria , including study design related quality assessment 
measures like clearly defined objective, standard data collection procedure, and statistical analysis plan. For each of the included studies, each criterion was rated either fulfilled (+), unfulfilled (-), or not applicable (NA). A quality score for each paper was obtained by counting the number of positives obtained; the score was then divided by 7 (minus the number of NAs) and multiplied by 100 , providing the percentage quality score. Studies scoring less than $60 \%$ were excluded for further review. Any discrepancy in quality assessment was discussed and internally resolved between 2 authors.

\section{Data Synthesis and Analysis}

As the primary outcome measure, we calculated the weighed pooled PR of NePC in patients with LBP. The weight, Wi, assigned here to each study was the inverse of the estimated variance. Where different prevalence estimates in the same patient group, using different diagnostic methods, were reported those prevalence estimates resulting from the most reliable method were chosen. For the present meta-analysis, the pooled PR, with $95 \% \mathrm{Cl}$, was reported as a summary measurement, with the results being presented as a forest plot. Cochran $Q$ and $I^{2}$ statistics were used to assess heterogeneity, with a $Q$ statistic $P$ value of $<0.10$, and an $I^{2}$ value $>$ $50 \%$ having been considered as statistically significant. If heterogeneity was identified, the DerSimonian-Laird random-effects model was applied; otherwise, the fixed effects-model (inverse variance) was considered (21). A pre-specified subgroup analysis was carried out to assess the source of heterogeneity, according to: i) diagnostic method(s) for identification of NeP (e.g., physician- or questionnaire-based), ii) duration of LBP (e.g., acute, subacute, or chronic), iii) presence of associated leg pain, iv) primary objective of the study being/not being the assessment of NeP prevalence, v) number of study centers involved (e.g., single vs multi-center), and vi) questionnaires used for the diagnosis (e.g., LANSS, DN4, PDQ, and NPQ). Tests for interaction using summary estimates were performed, using the method described by Altman and Bland (21).

To evaluate the stability of our results, we also performed a one-way sensitivity analysis. The scope of this analysis was to evaluate the influence of individual studies by estimating the average PR in the absence of each study. The present work was performed in accordance with the guidelines proposed by the Metaanalysis of Observational Studies in Epidemiology group and Preferred Reporting Items for Systematic Reviews and Meta-Analyses (PRISMA; supplementary material 1)
(22). The publication bias was assessed using both the funnel plot and the Begg's test. The Duval and Tweedie nonparametric trim-and-fill method was performed to further assess the effect of potential publication bias, a method which negotiates the lack of studies on a particular side of the funnel (23). When data were not uniformly reported to allow a formal statistical analysis, we presented the available data in a narrative format. All statistical tests were 2 -sided, and a $P$ value $<0.05$ was considered statistically significant, except where otherwise specified. Data were analyzed using STATA version 11.0 (StataCorp, College Station, TX, USA).

\section{Results}

\section{Search Results}

After applying the initial search strategy, a total of 160 citations were identified (Fig. 1). After reviewing the citations, 124 of them were considered ineligible, as they were reviews/editorials/case reports, hence not meeting the inclusion criteria. In examining the full text of the remaining 36 studies and reviewing the related reference lists, 5 further studies were considered. After a detailed evaluation of these 41 manuscripts, 21 of them were excluded for a range of reasons, which are better detailed in Fig. 1. As a result, 20 studies were included in the present meta-analysis.

\section{Description of Included Studies}

All the studies had been published between 2004 and 2015. Most ( $n=10 ; 50 \%)$ originated from Europe $(2,7,9,24-30)$, followed by Asia $(n=5 ; 25 \%)(10,17,31-$ $33)$, and Africa $(n=4 ; 20 \%)(8,34-36)$. The study sample recruitment site was not reported in one study (37).

Out of these studies, 17 (85\%) were cross sectional, 2 were cohort, and one was a case control study. Eleven (55\%) studies were multi-center $(2,10,24-27,29$ $31,34,35)$ whereas the remaining were single-center studies $(7-9,17,28,32,33,36,37)$. Twelve $(60 \%)$ studies were carried out primarily to assess the prevalence of NeP in patients with LBP, while the remaining focused either on the effects of $\mathrm{NeP}$ on a range of patientreported outcomes or on the validation of a range of NeP-related questionnaires. Eighteen (90\%) studies included patients with LBP as the main clinical concern, while 2 studies included patients with multiple pain conditions. The LBP etiology was reported in $8(40 \%)$ studies $(10,17,24,31,32,34-36)$.

All studies were carried out in hospital settings. Most $(n=16)$ studies had reported a diagnosis of 


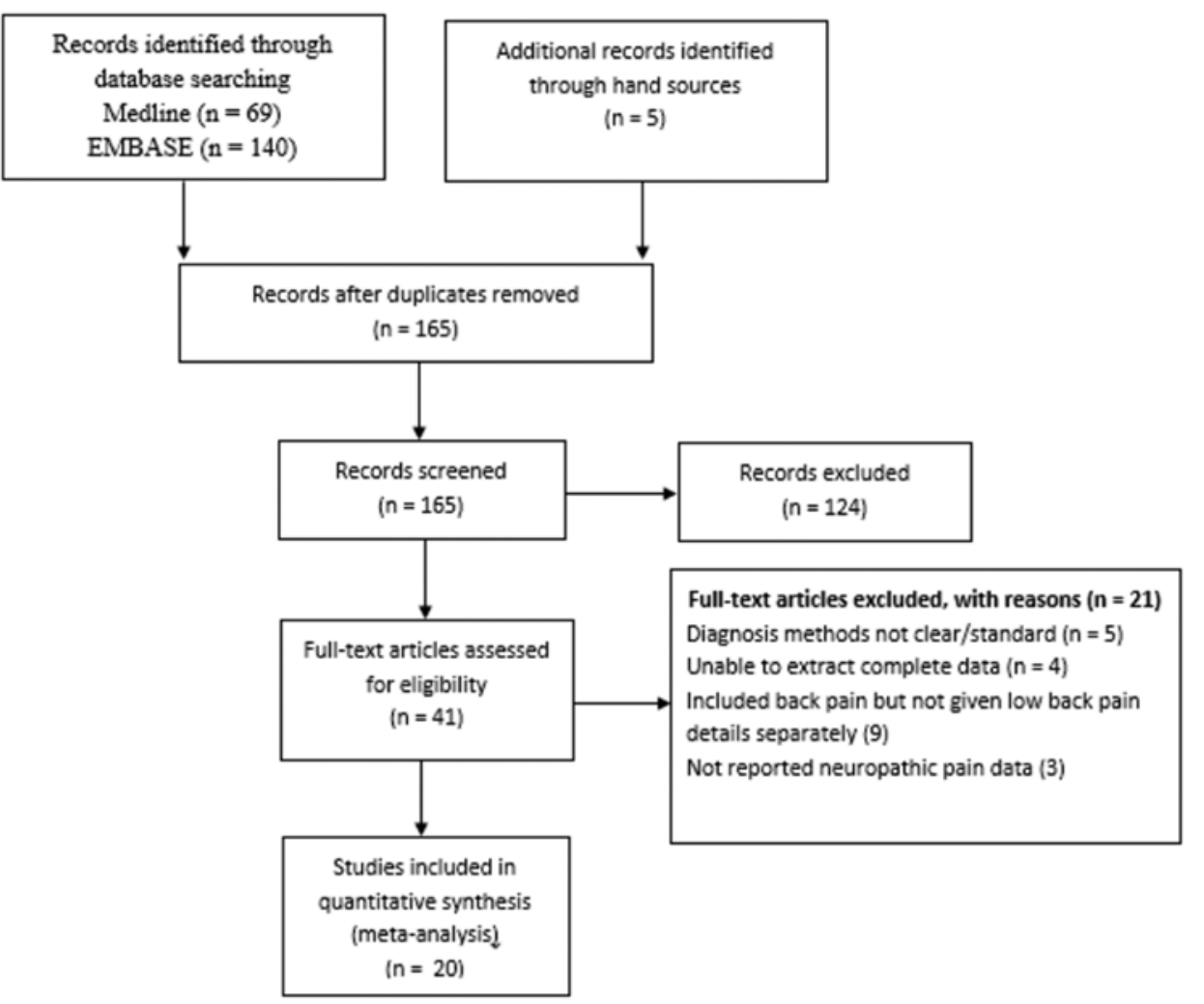

Fig. 1. Flow chart showing the number of citations retrieved by individual searches and the number of studies included in the review.

NePC which relied solely on symptom-based questionnaires, while the sole physician's criteria were used in 2 studies $(5,27)$. Only one study (7) reported an NePC diagnosis made with the help of both physician's criteria and symptom-based questionnaires. Most commonly used questionnaires included PDQ ( $n=7$ studies) $(2,9,17,25,26,28,33)$, followed by DN4 $(6,8,24,36,37)$, and LANSS $(6,31,32,34,35)(n=5$ each). Conversely, NPQ ( $n=2$ studies) $(10,33)$ and StEP ( $n=1$ study) (7) were less frequently considered (Table 1 ).

\section{Quality Assessment}

All the studies had a quality score ranging from $71 \%$ to $100 \%$, hence were eligible for inclusion. The average (SD) score was $92.8 \%$ (9.8\%) (Table 2). Two studies presented with a score of $71.4 \%$; in fact, they were not characterized by an adequate sample size (e.g., $n$ $<50$ patients) and had not considered a consecutive patient recruitment $(10,33)$.

\section{Primary Outcome}

As a significant heterogeneity was found between studies (Cochrane $\mathrm{Q} \leq 0.01, \mathrm{I}^{2}=98$ ), a random effects model was chosen (Table 3 ). The present meta-analysis included 14,269 patients with LBP, of whom 7,969 patients $(55.8 \%)$ were identified with NePC. The pooled PR $(95 \% \mathrm{Cl})$ of NePC in patients with LBP out of all the included studies was $0.47(0.40-0.54)$ (Fig. 2), with pooled PR for NePC being in the 0.28 to 0.71 range.

\section{Sensitivity Analysis}

The estimated effect size did not deviate much by excluding any study, one at a time, with pooled PR values ranging between 0.42 and 0.49 .

\section{Publication Bias}

The visual examination of the funnel plot revealed only minimal levels of asymmetry (Fig. 3). This was further confirmed by the Begg's test $(P=0.58)$ which indicated little or no publication bias. The NePC pooled 
Neuropathic Pain Component in Chronic Low Back Pain

Table 1. Characteristics of 20 studies included in the analysis to assess the prevalence rate of the neuropathic pain component in patients with low back pain.

\begin{tabular}{|c|c|c|c|c|c|c|c|}
\hline $\begin{array}{c}\text { Author, year (Reference } \\
\text { number of the study) }\end{array}$ & Country & $\begin{array}{l}\text { Method of } \\
\text { Diagnosis^ }^{\wedge}\end{array}$ & Questionnaire & $\begin{array}{c}\text { Sample } \\
\text { Size } \\
\end{array}$ & $\begin{array}{c}\text { Duration of } \\
\text { LBP\# } \\
\end{array}$ & $\begin{array}{l}\text { Prevalence } \\
(95 \% \text { CI })\end{array}$ & $\begin{array}{c}\text { Study } \\
\text { Quality } \%^{\$} \%\end{array}$ \\
\hline Hassan et al, 2004 (34) & SA & 1 & LANSS & 100 & Chronic & $41(31.4-50.6)$ & 83.3 \\
\hline Kaki et al, 2005 (35) & SA & 1 & LANSS & 1,169 & Chronic & $54.7(51.8-57.5)$ & 100 \\
\hline Freynhagen et al, 2006 (2) & Germany & 1 & PDQ & 7,772 & Chronic & $64.7(63.6-65.8)$ & 100 \\
\hline Freynhagen et al, 2006 (27) & Germany & 2 & - & 717 & Chronic & $33(29.6-36.4)$ & 100 \\
\hline Scholz et al, 2009 (7) & UK & $1,2^{*}$ & StEP & 137 & Chronic & $54.7(46.4-63)$ & 85.71 \\
\hline Sissi et al, 2010 (31) & UAE & 1 & LANSS & 1,134 & Chronic & $55.4(52.5-58.3)$ & 100 \\
\hline Attal et al, 2011 (24) & France & 1 & DN4 & 132 & Chronic & $70.4(62.6-78.2)$ & 100 \\
\hline Beith et al, 2011 (25) & $\begin{array}{l}\text { England, } \\
\text { UK }\end{array}$ & 1 & PDQ & 343 & NR & $40.5(35.3-45.7)$ & 85.71 \\
\hline Morsø et al, 2011 (28) & Denmark & 1 & PDQ & 145 & Chronic & $45.5(37.4-53.6)$ & 100 \\
\hline Ouedraogo et al, 2011 (36) & $\begin{array}{l}\text { Burkina } \\
\text { Faso, } \\
\text { Africa }\end{array}$ & 1 & DN4 & 107 & $\begin{array}{c}\text { Acute/Subacute, } \\
\text { Chronic }\end{array}$ & $49.5(40-59)$ & 100 \\
\hline \multirow{3}{*}{ Smart et al, 2012 (29) } & \multirow{3}{*}{$\begin{array}{l}\text { Ireland, } \\
\text { UK }\end{array}$} & \multirow{3}{*}{2} & \multirow{3}{*}{-} & 464 & $\begin{array}{l}\text { Acute/Subacute, } \\
\text { Chronic }\end{array}$ & $44.8(40.3-49.3)$ & \multirow{3}{*}{83.33} \\
\hline & & & & 123 & Acute/Subacute & $32.5(24.2-40.8)$ & \\
\hline & & & & 341 & Chronic & $49.3(44-54.6)$ & \\
\hline Walsh et al, $2012(30)$ & Ireland & 1 & S-LANSS, DN4* & 45 & NR & $42(27.6-56.4)$ & 85.71 \\
\hline \multirow{3}{*}{ Uher et al, 2012 (9) } & \multirow{3}{*}{$\begin{array}{l}\text { Czech } \\
\text { Republic }\end{array}$} & \multirow{3}{*}{1} & \multirow{3}{*}{ PDQ } & 66 & $\begin{array}{c}\text { Acute/Subacute, } \\
\text { Chronic }\end{array}$ & $71.2(60.3-82.1)$ & \multirow{3}{*}{100} \\
\hline & & & & 40 & Acute/Subacute & $70(55.8-84.2)$ & \\
\hline & & & & 26 & Chronic & $73(56-90.1)$ & \\
\hline Yamashita et al, 2013 (10) & Japan & 1 & NPQ & 17 & Chronic & $29.4(7.7-51)$ & 66.66 \\
\hline Forster et al, 2013 (26) & Germany & 1 & PDQ & 1,083 & NR & $30.7(28-33.4)$ & 83.33 \\
\hline Selimoglu et al, 2013 (37) & NR & 1 & DN4 & 224 & Chronic & $55.8(49.3-62.3)$ & \\
\hline Doualla et al, 2013 (8) & Cameroon & 1 & DN4 & 167 & Chronic & $28.1(21.3-35)$ & \\
\hline \multirow{3}{*}{ Hiyama et al, 2014 (17) } & \multirow{3}{*}{ Japan } & \multirow{3}{*}{1} & \multirow{3}{*}{ PDQ } & 331 & $\begin{array}{l}\text { Acute/Subacute, } \\
\text { Chronic }\end{array}$ & $42.5(37.2-47.8)$ & \multirow{3}{*}{100} \\
\hline & & & & 201 & Acute/Subacute & $25.3(19.3-31.3)$ & \\
\hline & & & & 130 & Chronic & $31.5(23.5-39.5)$ & \\
\hline Sakai et al, 2015 (33) & Japan & 1 & $\mathrm{NPQ}^{*}, \mathrm{PDQ}$ & 30 & Chronic & $43.3(25.6-61)$ & 66.66 \\
\hline Park et al, 2015 (32) & Korea & 1 & LANSS & 86 & NR & $36(25.8-46.1)$ & 100 \\
\hline
\end{tabular}

NR, Not reported; SA, South Africa; UK, United Kingdom; UAE, United Arab Emirates; LANSS, Leeds Assessment of Neuropathic Symptoms and Signs; NPQ, Neuropathic Pain Questionnaire; PDQ, PainDETECT Questionnaire; DN4, Douleur Neuropathique 4 questionnaire

$\wedge$ : questionnaire; 2 : clinician rated ${ }^{*}$ Standard method considered if more than one method used for the diagnosis of NPC

\# Acute: duration of LBP less than 6 weeks; Subacute: duration of LBP 6 to 12 weeks; Chronic: duration of LBP more than 12 weeks.

C.I; Confidence Interval

${ }^{\mathrm{s}}$ Quality was assessed using scale developed by Fishbain et al (here modified)

PR value (e.g., 0.47; 0.40 - 0.54) obtained using the random effects model remained unchanged using the Duval and Tweedie's (23) trim-and-fill analysis.

\section{Sub-group Analysis}

With the physician-diagnosis approach, the pooled
PR of NePC in LBP patients was lower $(0.44 ; 0.32-0.55)$ than the pooled PR resulting from the questionnairebased diagnosis $\left(0.48 ; 0.40-0.55 ; P_{\text {interaction }}=0.58\right)$. Similarly, the pooled PR of NePC was only marginally higher (0.49; $0.41-0.57)$ in CLBP patients in comparison with patients affected by acute/subacute LBP $(0.45 ; 0.28$ - 


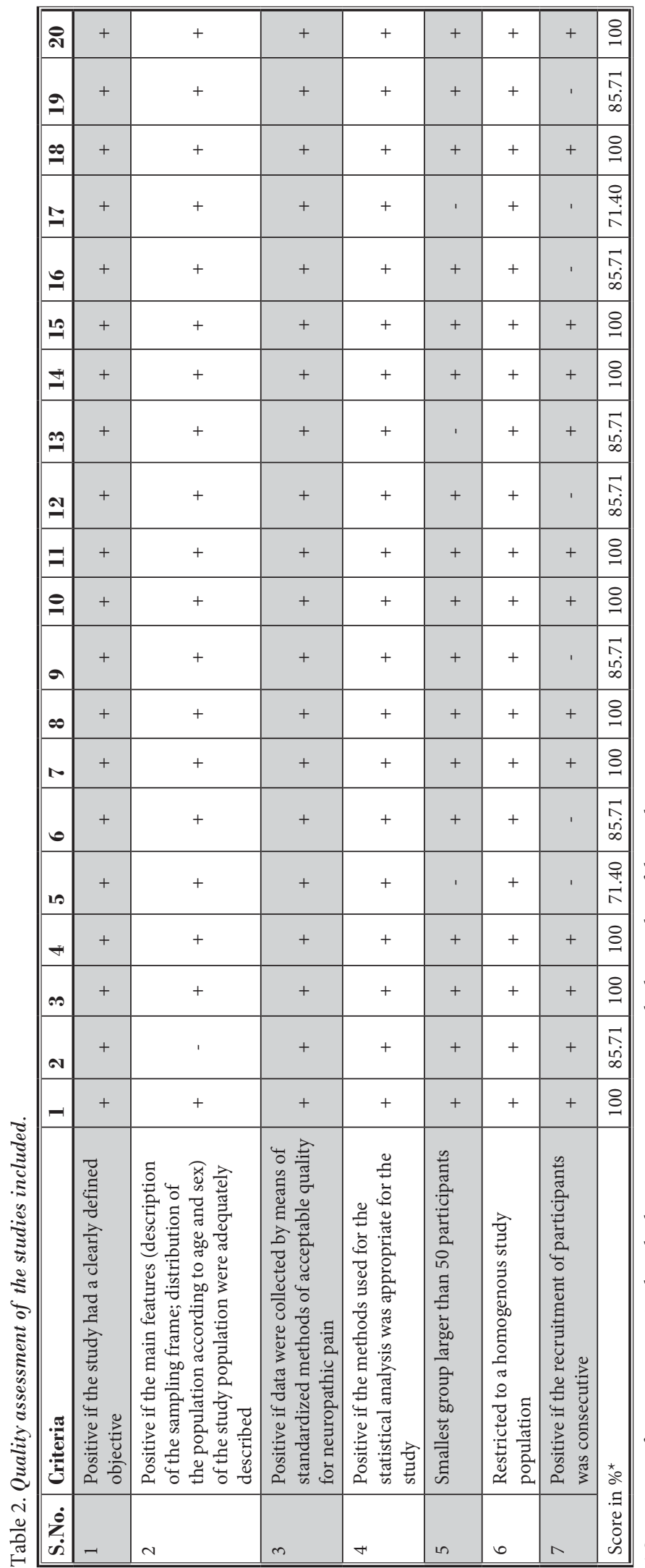

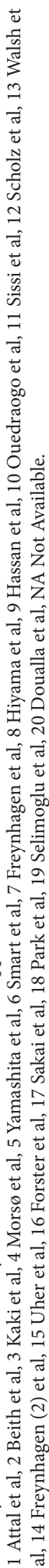

0.62; $\left.P_{\text {interaction }}=0.71\right)$. Conversely, significantly higher pooled PR values of NePC were associated with leg pain $(0.60 ; 0.47$ - 0.73) as compared to those conditions without an associated leg pain (0.27; $\left.0.23-0.31 ; P_{\text {interaction }}<0.01\right)$. When the subgroup analysis was performed according to the type of screening questionnaires used, comparable PR were obtained with LANSS $(0.47 ; 0.29-0.64)$, DN4 (0.49; $0.33-0.65)$, and PDQ (0.48; $0.34-0.63)$. However, relatively lower $\mathrm{PR}$ values of $\mathrm{NePC}$ were identified when the NPQ was used $(0.36 ; 0.90-0.64)$.

\section{Secondary Outcomes}

The pooled PR of mixed pain in patients with LBP ( $n=7$ studies) was found to be $0.28(0.23-0.34)$ (Table 4$)$, while the pooled PR of sole NeP in patients with LBP ( $n=7$ studies) was found to be 0.23 (0.11-0.34). The pooled PR of NcP in patients with LBP in all studies was $0.56(0.48-0.63)$.

\section{Discussion}

The present meta-analysis of 20 studies, including 14,269 participants, found that the PPR of NePC in patients with LBP was $47 \%(40 \%-54 \%)$, hence NePC may need to be considered as a significant clinical problem in these patients. One of the major strengths of the current study is that we focused on both the neuropathic and the nociceptive components, while considering not only the chronic, but also the acute/ subacute, LBP conditions. Moreover, previous studies considered mathematic calculations only to estimate NePC pooled PR values, as opposed to the formal meta-analysis approach which was carried out here. The NePC figure $(47 \%)$ identified is consistent with previous findings, e.g., Morso et al (45.5) and Ouedraogo et al $(49.5)(28,36)$, but it is at odds with the figures reported by both Fishbain et al (36.6\%) and Mehra et al $(90.4 \%)(20,38)$. The studies here reported a prevalence of NePC ranging from $28.1 \%$ to $71.2 \%$, with these levels 
Neuropathic Pain Component in Chronic Low Back Pain

Table 3. Pooled prevalence rates of NePC in different subgroups of patients with LBP.

\begin{tabular}{|c|c|c|c|c|c|c|c|c|}
\hline \multirow[b]{2}{*}{ Study Characteristics } & & \multicolumn{4}{|c|}{ Neuropathic pain component } & \multicolumn{3}{|c|}{ Heterogeneity } \\
\hline & & Studies & $\begin{array}{c}\text { No. of } \\
\text { Patients }\end{array}$ & $\begin{array}{c}\text { PR } \\
(95 \% \text { CI })\end{array}$ & $P$ value & Cochrane Q & $\mathbf{I}^{2}$ & $P$ Subgroup\# \\
\hline All studies & 14269 & 20 & 7969 & $0.47(0.40-0.54)$ & $<0.01$ & $<0.01$ & 98.0 & NA \\
\hline \multicolumn{9}{|l|}{ Leg pain } \\
\hline Present & 572 & 5 & 300 & $0.60(0.47-0.73)$ & $<0.01$ & $<0.01$ & 89.9 & \multirow{2}{*}{$<0.01$} \\
\hline Absent & 479 & 2 & 89 & $0.27(0.23-0.31)$ & $<0.01$ & $<0.01$ & 97.5 & \\
\hline \multicolumn{9}{|l|}{ Diagnostic method } \\
\hline Physician-based & 1318 & 3 & 520 & $0.44(0.32-0.55)$ & $<0.01$ & $<0.01$ & 93.6 & \multirow{2}{*}{0.58} \\
\hline Questionnaire-based & 12951 & 17 & 4792 & $0.48(0.40-0.55)$ & $<0.01$ & $<0.01$ & 97.9 & \\
\hline \multicolumn{9}{|l|}{ Duration of pain } \\
\hline Acute/Subacute & 364 & 3 & 80 & $0.45(0.28-0.62)$ & $<0.01$ & $<0.01$ & 90.5 & \multirow{2}{*}{0.71} \\
\hline Chronic & 51666 & 15 & 40585 & $0.49(0.41-0.57)$ & $<0.01$ & $<0.01$ & 97.6 & \\
\hline \multicolumn{9}{|l|}{ Study Centres } \\
\hline Single center & 1293 & 09 & 598 & $0.47(0.39-0.55)$ & $<0.01$ & $<0.01$ & 87.8 & \multirow{2}{*}{0.53} \\
\hline Multi-center & 12976 & 11 & 7371 & $0.46(0.37-0.56)$ & $<0.01$ & $<0.01$ & 98.8 & \\
\hline \multicolumn{9}{|c|}{ Primary objective was to assess the prevalence of NePC in LBP } \\
\hline Yes & 5267 & 12 & 2373 & $0.44(0.36-0.52)$ & $<0.01$ & $<0.01$ & 96.6 & \multirow{2}{*}{0.57} \\
\hline No & 9002 & 08 & 5596 & $0.51(0.41-0.60)$ & $<0.01$ & $<0.01$ & 96.1 & \\
\hline \multicolumn{9}{|l|}{ Questionnaire used } \\
\hline LANSS & 2489 & 4 & 1339 & $0.47(0.29-0.64)$ & $<0.01$ & $<0.01$ & 85.1 & \\
\hline DN4 & 675 & 5 & 337 & $0.49(0.33-0.65)$ & $<0.01$ & $<0.01$ & 94.2 & \\
\hline PDQ & 9740 & 6 & 5755 & $0.48(0.34-0.63)$ & $<0.01$ & $<0.01$ & 99.1 & \\
\hline NPQ & 47 & 2 & 18 & $0.36(0.90-0.64)$ & $<0.01$ & $<0.01$ & 0.00 & \\
\hline
\end{tabular}

Comparison between subgroups

NA, Not Available; CI, Confidence Interval; PR, Prevalence Rate; LANSS, Leeds Assessment of Neuropathic Symptoms and Signs; NPQ, Neuropathic Pain Questionnaire; NePC, Neuropathic pain component; PDQ, PainDETECT Questionnaire; DN4, Douleur Neuropathique 4 questionnaire; LBP, low back pain.

of variations possibly being explained by differences in inclusion criteria, sample size, study settings, and diagnostic methods used $(8,9)$. Furthermore, some diagnostic questionnaires fail to identify the mixed pain conditions, hence underestimating the presence of $\mathrm{NeP}$ symptoms $(31,35,36)$. According to the current sensitivity analysis, excluding any single study did not have here any major impact on the combined results, which may well suggest that the present meta-analysis results are pretty consistent. Furthermore, both a robustness of results and lack of publication bias were confirmed.

Overall, a physician/clinician decision is considered to be the gold standard in diagnosing any type of pain, with the diagnostic accuracy of screening questionnaires not always being considered satisfactory (39). However, similar NePC prevalence levels were identified in those studies using questionnaires $(0.48 ; 0.40-0.55)$ vs those considering the clinician-based diagnosis $(0.44$; $0.32-0.55)$.

LBP duration was hypothesized to be a possible factor associated with different NePC prevalence levels. However, the NePC PPR value was only marginally higher in patients with CLBP than in those experiencing acute/subacute LBP (respectively: 0.49; $0.41-0.57$; vs $0.45 ; 0.28-0.62 ; n s$ ). Indeed, acute and chronic LBP cases differ in their etiology and pathophysiology, with acute LBP being usually a self-limiting, protective, biological function of on-going tissue damage. Acute pain is mostly nociceptive in origin and occurs secondary to stimulation of A-delta and C-polymodal pain receptors (16). Conversely, CLBP is in itself a disease process which can persist for years after the initial injury and which can be refractory to multiple treatment modalities. Inadequately treated acute pain conditions may be asso- 


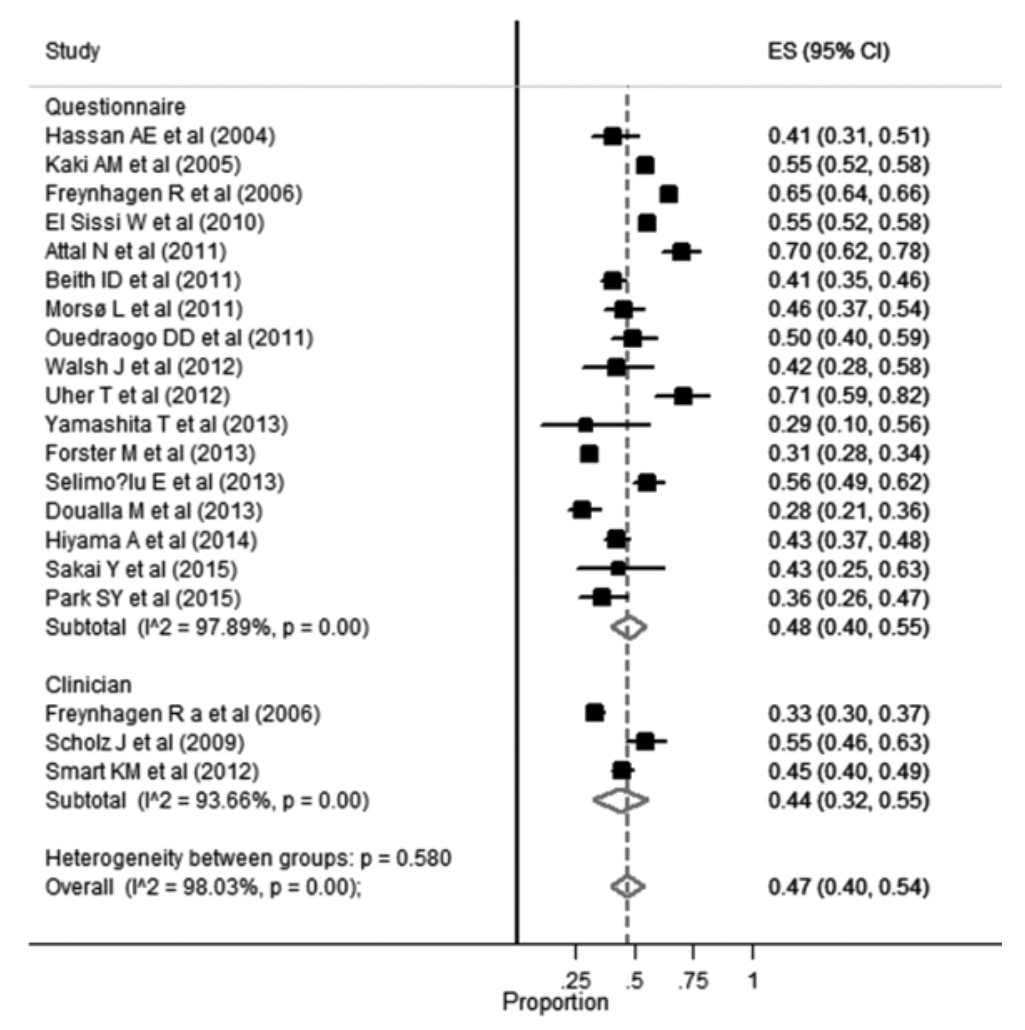

NPC, neuropathic pain component; LBP, low back pain; CI, confidence interval.

Fig. 2. Pooled estimate of prevalence rate and $95 \%$ CIs of NPC in patients with $L B P$ in all the included studies (20 studies) comprising 14,269 patients with LBP. The pooled prevalence rate of NPC in patients with LBP in all the included studies (20 studies) was found to be 0.47 (at 95\% CI, 0.40-0.54).

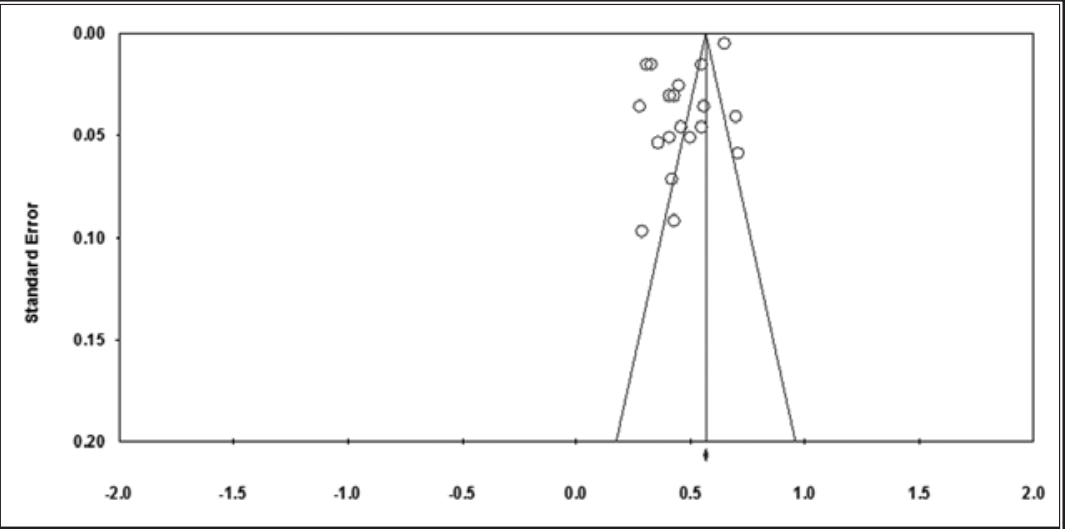

Fig. 3. Funnel plot to assess the publication bias (effect estimates on the horizontal scale and study size on the vertical axis). ciated with NeP symptoms (16) and may become chronic. Consistent with this, a higher prevalence of NePC is typically identified in CLBP as opposed to acute/subacute LBP (17).

The presence of leg pain was hypothesized as another possible factor associated with different NePC prevalence levels. As expected, and consistent with previous findings (17), the NePC prevalence was significantly higher in patients affected by leg pain than in those with uncomplicated LBP. Although both $\mathrm{NeP}$ and NcP mechanisms are thought to contribute to leg pain in LBP, neuropathic mechanisms play a more significant role $(1,27)$ Evidence also suggests that patients experiencing a radiating leg pain may complain of intense pain and significant disability (40), delayed recovery, and a tendency to chronicity (41). Furthermore, they frequently require surgical interventions for their LBP to be managed (42). To better identify the presence of NePC in LBP, some questionnaires (e.g., PDQ) have been designed to specifically include a question about the presence of leg pain (12). Results of the present meta-analysis might not be generalizable to patients with failed back syndrome where the prevalence of $\mathrm{NeP}$ is more common and the cause for NeP is quite different.

\section{Limitations}

The quality of the studies selected was assessed using an ad hoc, customized scale. Although not specifically validated, the scale presented has been designed while including further assessment criteria to previous instruments. Due to the limited number of available 
studies, one may need to be cautious in concluding about the impact of disease duration on NePC prevalence values. Furthermore, the quality of data collected from those studies whose primary aim was not the assessment of $\mathrm{NeP}$ prevalence may be questionable. Similarly, one could wonder about the appropriateness of grouping together studies which used a range of different diagnostic methods, with putatively different sensitivity/specificity diagnosing levels. To address this issue, however, a subgroup analysis based on type of questionnaires used was carried out. The limitation of current diagnostic methods is that we cannot quantify how much neuropathic there is vs. nociceptive to direct the clinician to the appropriate therapy. The studies included did not present with sufficient data to perform a subgroup analysis to estimate NeP prevalence levels according to specific clinical conditions/pathologies involved. Finally, only a few studies included patients with LBP with an associated leg pain condition $(9,28,30)$, so that the results presented may need to be interpreted with some caution. There are limited studies from the US.

Given the difficulties in treating and managing clients affected by low back pain with a neuropathic component further research studies with larger sample sizes and increased diagnostic accuracy levels should be promoted. As pain is subjective phenomenon and as there is a lack of a gold standard for the diagnosis of $N P$, there is the possibility that the pooled effect estimate may alter if the diagnostic ability of the available and used methods warrant the gold accuracy.

\section{References}

1. Freynhagen R, Baron R. The evaluation of neuropathic components in low back pain. Curr Pain Headache Rep 2009; 13:185-190.

2. Freynhagen R, Baron R, Gockel U, Tolle $T$. painDETECT: A new screening questionnaire to detect neuropathic components in patients with back pain. Curr Med Res Opin 2006; 22:1911-1920.

3. Krause SJ, Backonja MM. Development of a neuropathic pain questionnaire. Clin J Pain 2003; 19:306-314.

4. Bennett MI. The LANSS Pain Scale: The Leeds Assessment of Neuropathic Symptoms and Signs. Pain 2001; 92:147-157.

5. Portenoy R. Development and testing of a neuropathic pain screening questionnaire: ID Pain. Curr Med Res Opin 2006; 22:1555-1565.

6. Bouhassira D, Attal $\mathrm{N}$, Alchaar $\mathrm{H}$, Boureau F, Brochet B, Bruxelle J, Cunin G, Fermanian J, Ginies P, GrunOverdyking A, Jafari-Schluep $\mathrm{H}$, LantériMinet M, Laurent B, Mick G, Serrie A, Valade $D$, Vicaut $E$. Comparison of pain syndromes associated with nervous or somatic lesions and development of a new neuropathic pain diagnostic questionnaire (DN4). Pain 2005; 114:29-36.

7. Scholz J, Mannion RJ, Hord DE, Griffin RS, Rawal B, Zheng H, Scoffings D, Phillips A, Guo J, Laing RJ, Abdi S, Decosterd I, Woolf CJ. A novel tool for the assessment of pain: Validation in low back pain. PLoS Med 2009; 6:e1000047.
8. Doualla M, Luma HN, Tchaleu BN, Kwedi, Kemta FL, Memopi M. The neuropathic component of chronic low back pain in Douala-Cameroon. Clin Rheumatol 2013; 32:S123-S124.

9. Uher T, Bob P Neuropathic pain, depressive symptoms, and C-reactive protein in sciatica patients. Int J Neurosci 2013; 123:204-208.

10. Yamashita T, Takahashi K, Yonenobu K Kikuchi S. Prevalence of neuropathic pain in cases with chronic pain related to spinal disorders. J Orthop Sci 2014; 19:15-21.

11. Froud R, Patterson S, Eldridge S, Seale $C$, Pincus T, Rajendran D, Fossum C, Underwood M. A systematic review and meta-synthesis of the impact of low back pain on people's lives. BMC Musculoskelet Disord 2014; 15:50.

12. Treede RD, Jensen TS, Campbell JN, Cruccu G, Dostrovsky JO, Griffin JW, Hansson $P$, Hughes R, Nurmikko T, Serra J. Redefinition of neuropathic pain and a grading system for clinical use: Consensus statement on clinical and research diagnostic criteria. Neurology 2008; 70:1630-1635.

13. Sörensen J, Aaro S, Bengtsson M, Kalman S, Reigo T, Tropp H. Can a pharmacological pain analysis in patients with chronic low back pain predict the outcome of lumbar fusion? Preliminary report. Eur Spine J 1996; 5:326-331.

14. Morlion B. Pharmacotherapy of low back pain: Targeting nociceptive and neuro- pathic pain components. Curr Med Res Opin 2011; 27:11-33.

15. Kalita J, Kohat AK, Misra UK, Bhoi SK. An open labeled randomized controlled trial of pregabalin versus amitriptyline in chronic low backache. J Neurol Sci 2014; 342:127-132.

16. National Pain Strategy. Pain Management for all Australians. Available at: www.iasp-pain.org/files/Content/NavigationMenu/Advocacy/InternationalPainSummit/Australia_2010PainStrategy. pdf. Date of Access: 23/05/2016

17. Hiyama A, Watanabe $M$, Katoh $H$, Sato M, Sakai D, Mochida J. Evaluation of quality of life and neuropathic pain in patients with low back pain using the Japanese Orthopedic Association Back Pain Evaluation Questionnaire. Eur Spine J 2015; 24:503-512.

18. Hoogendoorn WE, Van Poppel MN, Bongers PM, Koes BW, Bouter LM. Systematic review of psychosocial factors at work and private life as risk factors for back pain. Spine 2000; 25:2114-2125.

19. De Vet H, De Bie R, van der Heijden G, Arianne P Verhagen, Petra Sijpkes, Paul $G$ Knipschild. Systematic review of basis of methodological criteria. Physiotherapy 1997; 83:284-289.

20. Fishbain DA, Cole B, Lewis JE, Gao J. What is the evidence that neuropathic pain is present in chronic low back pain and soft tissue syndromes? An evidencebased structured review. Pain Med 2014; 15:4-15. 
21. Higgins JP, Thompson SG, Deeks JJ, Altman DG. Measuring inconsistency in meta-analyses. BM] 2003; 327:557-560.

22. Moher D, Liberati A, Tetzlaff J, Altman DG, PRISMA Group: Preferred reporting items for systematic reviews and meta-analyses: The PRISMA Statement. BM] 2009; 339:b2535.

23. Duval S, Tweedie R. Trim and fill: A simple funnel-plot based method of testing and adjusting for publication bias in meta-analysis. Biometrics 2000; 56:455-463.

24. Attal N, Perrot S, Fermanian J, Bouhassira $\mathrm{D}$. The neuropathic components of chronic low back pain: A prospective multicenter study using the $\mathrm{DN}_{4}$ Questionnaire. J Pain 2011; 12:1080-1087.

25. Beith ID, Kemp A, Kenyon J, Prout M, Chestnut TJ. Identifying neuropathic back and leg pain: A cross-sectional study. Pain 2011; 152:1511-1516.

26. Förster M, Mahn F, Gockel U, Brosz M, Freynhagen R, Tölle TR, Baron R. Axial low back pain: one painful area - many perceptions and mechanisms. PLoS One 2013; 8:e68273.

27. Freynhagen R, Baron R, Tölle T, Stemmler E, Gockel U, Stevens M, Maier C. Screening of neuropathic pain components in patients with chronic back pain associated with nerve root compression: A prospective observational pilot study (MIPORT). Curr Med Res Opin 2006; 22:529-537.

27. Morsø L, Kent PM, Albert HB. Are selfreported pain characteristics, classified using the PainDETECT questionnaire, predictive of outcome in people with low back pain and associated leg pain? Clin J Pain 2011; 27:535-541.

28. Smart KM, Blake C, Staines A, Doody C. Self-reported pain severity, quality of life, disability, anxiety and depression in patients classified with 'nociceptive,' 'peripheral neuropathic' and 'central sensitisation' pain. The discriminant validity of mechanisms-based classifications of low back ( \pm leg) pain. Man Ther 2012; 17:119-125.

29. Walsh J, Rabey MI, Hall TM. Agreement and correlation between the self-report Leeds Assessment of Neuropathic Symptoms and Signs and Douleur Neuropathique 4 Questions neuropathic pain screening tools in subjects with low back-related leg pain. J Manipulative Physiol Ther 2012; 35:196-202.

30. El Sissi W, Arnaout A, Chaarani MW, Fouad M, El Assuity W, Zalzala M, Dershaby YE, Youseif E. Prevalence of neuropathic pain among patients with chronic low-back pain in the Arabian Gulf Region assessed using the Leeds assessment of neuropathic symptoms and signs pain scale. J Int Med Res 2010; 38:2135-2145.

31. Park SY, An HS, Moon SH, Lee HM, Suh SW, Chen D, Jeon JH. Neuropathic pain components in patients with lumbar spinal stenosis. Yonsei Med J 2015; 56:1044-1050.

32. Sakai $Y$, Ito $K$, Hida $T$, Ito $S$, Harada A. Neuropathic pain in elderly patients with chronic low back pain and effects of pregabalin: A preliminary study. Asian Spine J 2015; 9:254-262.

33. Hassan AE, Saleh HA, Baroudy YM, Abdul-Rahman KI, Najjar MW, Kazi MS, El-Gazar MA, Hafez MA, Abdullah MA, Abdul-Rahman YA, Youseif EA. Prevalence of neuropathic pain among patients suffering from chronic low back pain in Saudi Arabia. Saudi Med J 2004; 25:1986-1990.

34. Kaki AM, El-Yaski AZ, Youseif E. Identifying neuropathic pain among patients with chronic low-back pain: Use of the Leeds Assessment of Neuropathic Symptoms and Signs pain scale. Reg Anesth Pain Med 2005; 30:422-428.

35. Ouédraogo DD, Nonguierma $V$, Napon C, Kabré A, Tiéno H, Guira O, Kaboré J, Drabo JY. Prevalence of neuropathic pain among Black African patients suffering from common low back pain. Rheumatol Int 2012; 32:2149-2153.

36. Selimoglu E, Murat S, Turgut ST, Gürek SY, Icagasioglu A. The prevelance of neuropathic pain in patients with chronic low back pain and its relationships with quality of life, disability and depression. The Turkish Journal of Physical Medicine and Rehabilitation 2013; 59:293.

37. Mehra M, Hill K, Nicholl D, Schadrack J. The burden of chronic low back pain with and without a neuropathic component: $A$ healthcare resource use and cost analysis. J Med Econ 2012; 15:245-252.

38. Mathieson S, Maher CG, Terwee CB, de Campos TF, Lin CW. Neuropathic pain screening questionnaires have limited measurement properties. A systematic review. J Clin Epidemiol 2015; 68:957-966.

39. Dunn KM, Croft PR. Epidemiology and natural history of low back pain. Eura Medicophys 2004; 40:9-13.

40. Mehling WE, Gopisetty V, Bartmess E, Acree M, Pressman A, Goldberg H, Hecht FM, Carey T, Avins AL. The prognosis of acute low back pain in primary care in the United States: A 2-year prospective cohort study. Spine 2012; 37:678-684

41. Verwoerd AJ, Luijsterburg PA, Lin CW, Jacobs WC, Koes BW, Verhagen AP. Systematic review of prognostic factors predicting outcome in non-surgically treated patients with sciatica. Eur J Pain 2013; 17:1126-1137. 